\section{Dilemas del personal médico ante la violencia de pareja en México}

\author{
Dilemmas of healthcare providers towards \\ domestic violence in Mexico
}

\begin{abstract}
1 Centro de Investigación en Sistemas en Salud, Instituto Nacional de Salud Pública, Cuernavaca, México.

2 Centro Regional

de Investigaciones Multidisciplinarias, Universidad Nacional Autónoma de México, México DF, México.

Correspondencia C. Agoff

Centro Regional

de Investigaciones

Multidisciplinarias,

Universidad Nacional

Autónoma de México.

Real de los Reyes 87 ,

A-403, Los Reyes

Coyoacán, 04330 México DF agoff@correo.crim.unam.mx
\end{abstract}

\section{Abstract}

This qualitative study of 30 physicians and nurses from the Mexican public health system aimed to understand their concepts and practices concerning domestic violence. A recent survey showed that most health professionals were unaware of the official criteria and procedures for dealing with cases of domestic violence. Our in-depth interviews failed to show substantial differences between professionals according to level of care, and that treatment was generally inadequate. We also observed a repertoire of ingrained social meanings among the medical staff concerning violence (derived from their own cultural traditions), hindering more effective responses to the problem.

Domestic Violence; Health Personnel; Violence Against Women
Cristina Herrera ${ }^{1}$

Carolina Agoff 2

\section{Introducción}

En 1993, la Asamblea General de las Naciones Unidas 1 definió la violencia contra las mujeres como: "todo acto de violencia basada en la pertenencia al sexo femenino, que tenga o pueda tener como resultado un daño o sufrimiento físico, sexual o psicológico para la mujer, así como las amenazas de tales actos, la coerción o la privación arbitraria de la libertad, tanto si se producen en la vida pública como en la vida privada". Como consta en la fecha de esta declaración, el maltrato a las mujeres en el interior de la familia no es caracterizado como "violencia" sino hasta muy recientemente. Asimismo, en 1996, la 49a Asamblea Mundial de la Salud 2 en su Resolución WHA49.25 reconoció la prevención de la violencia como una prioridad de salud pública y adoptó las recomendaciones formuladas dos años antes en la Conferencia Internacional sobre Población y Desarrollo ${ }^{3}$ de El Cairo, Egipto, y las de la Cuarta Conferencia Mundial sobre la Mujer 4 de Pekín, China, en 1995. Así, a partir de la última década del siglo XX el problema de la violencia familiar ha sido objeto de creciente discusión pública, escrutinio científico y diseño de políticas destinadas a su erradicación.

En el caso de México, la Secretaría de Salud junto con el Instituto Nacional de Salud Pública llevaron a cabo en el año 2002 la primera Encuesta Nacional de Violencia contra las Mujeres 
entre usuarias de servicios de salud pública (ENVIM) 5 y revelaron que de una muestra de 26.240 entrevistadas el $21,5 \%$ de las mujeres había padecido algún tipo de violencia en el hogar por parte de su pareja en los doce meses previos a la entrevista. El propósito de esta encuesta fue conocer la prevalencia y características de la violencia masculina contra las mujeres, como también la percepción y accionar del personal de salud. El grupo profesional de los prestadores de salud constituye uno de los actores clave para la identificación, notificación y canalización de los casos de violencia intrafamiliar hacia instancias de resolución jurídica. La caracterización de la violencia contra las mujeres, como problema de salud pública en México, y por tanto como un objeto de políticas y programas gubernamentales, tiene algunos de sus antecedentes en la Norma Oficial Mexicana Prestación de Servicios de Salud. Criterios para la Atención Médica de la Violencia Familiar (NOM-190) 6, que se creó en 1999 para "dar cumplimiento a los compromisos adquiridos en los foros internacionales en materia de la eliminación de todas las formas de violencia, especialmente la que ocurre en el seno de la familia y contra la mujer". Esta norma establece los criterios para la atención médica y la orientación de las y los usuarios que se encuentren involucrados en situaciones de violencia familiar y es de observancia obligatoria para todos los prestadores de servicios de salud de los sectores público, social y privado que componen el Sistema Nacional de Salud. Entre otros rasgos característicos, la NOM-190 provee una serie de indicadores para detectar distintos tipos de violencia (física, sexual, psicológica, económica), establece que esto debe ser llevado a cabo con oportunidad, calidez, confidencialidad, honestidad, y respeto a la dignidad, y al mismo tiempo establece la obligación de dar aviso al Ministerio Público (primera instancia del sistema judicial) y de notificar a la Secretaría de Salud sobre los casos de violencia familiar detectados. El apartado de criterios específicos, que corresponde a la notificación al sistema de vigilancia epidemiológica de la Secretaría de Salud, deja bien establecido el carácter obligatorio de este registro. Sin embargo, los criterios para dar aviso al Ministerio Público dejan espacio a interpretaciones ambiguas sobre en qué casos y quién debe involucrarse en denuncias.

La aplicación práctica de estas normas presenta múltiples dificultades. En la mencionada ENVIM 5, se incluyó un cuestionario de autoaplicación voluntaria para todo el equipo de salud, al que respondieron un total de 2.638 trabajadores. Del personal encuestado sólo 18,5\% refirió conocer la NOM-190. De ellos, 42,2\% conocía bien su contenido y el resto sólo había oído hablar de ella. Pero además, $32,8 \%$ de los entrevistados consideró que el desinterés del personal de salud por los casos de violencia contra la mujer era el principal obstáculo para su atención, y $22 \%$ opinó que la violencia en la familia era un problema privado y que al personal de salud no le correspondía intervenir en esos casos. Es importante señalar que $10,8 \%$ de los hombres y $16,9 \%$ de las mujeres que contestaron esta encuesta declaró ser víctima de violencia por parte de su pareja y que $12,2 \%$ de los hombres y $9,7 \%$ de las mujeres, que contestaron, había sido agresor(a) de su pareja. También llama la atención el hecho que $91,2 \%$ de estos profesionales se declaró interesado en capacitarse sobre el tema de violencia familiar y contra la mujer.

Para indagar en posibles explicaciones de estas aparentes contradicciones entre la normatividad, las prácticas profesionales y las propias opiniones de los prestadores de salud, entre 2003 y 2004 se llevó a cabo un estudio cualitativo que tuvo por objetivo profundizar en los motivos por los cuales estos profesionales no estaban respondiendo a los requerimientos del sector salud y de la normatividad legal respecto del problema de la violencia contra las mujeres. El estudio fue motivado por la convicción de que un análisis de las prácticas y significados de la violencia doméstica por parte de los profesionales indicados para atenderla y notificarla, aportaría elementos útiles para la discusión sobre la capacidad de las instituciones de salud para responder a un problema viejo, pero cuya "emergencia" y re-significación podrían traducirse en un incremento en la demanda a las mismas.

\section{Métodos}

Se diseñó un estudio cualitativo en el que se realizaron 30 entrevistas en profundidad con médicos/as y enfermeros/as de las tres instituciones públicas del sector salud en México (asegurados privados, asegurados públicos y población abierta), en tres entidades federativas con alta prevalencia de violencia de pareja (Quintana Roo, Coahuila y el Distrito Federal) de acuerdo con la ENVIM 5. De esas entrevistas, 16 fueron con médicos/as y 14 con enfermeros/as. También se realizó observación participante en los servicios de salud y diario de campo en las tres entidades y entrevistas con informantes clave (autoridades del sector, directores/as de las unidades de atención, jefes de programas 
especiales y de servicios). El propósito fue indagar a través de una guía de preguntas abiertas los siguientes aspectos: sensibilidad ante el tema de la violencia de pareja y calidad de la atención que brindan a la mujer maltratada; canalización de casos a otras instancias y disposición para atestiguar en un proceso legal; conocimiento y aplicación de la NOM-190; concepciones sobre la violencia de pareja, sus causas y consecuencias; capacitación recibida sobre violencia de pareja; y posibilidad de brindar atención y apoyo para las usuarias que quisieran abortar legalmente en caso de violación (el análisis de este punto no se incluye en el presente artículo). A través de este guión de preguntas se pretendió identificar los factores sociales que dan sustento a las prácticas observadas o reportadas. Las entrevistas tuvieron una duración promedio de 50 minutos y fueron conducidas y audio-grabadas por un equipo de cuatro investigadores (dos psicólogas, un psicólogo y una socióloga). El análisis de los datos se llevó a cabo según los principios de la teoría fundamentada, y consiste básicamente en la elaboración de hipótesis emergentes a partir de los datos y en su constatación mediante el estudio comparativo de casos seleccionados en virtud de su valor informativo 7,8. El protocolo de investigación fue aprobado por el Comité de Evaluación Ética del Instituto Nacional de Salud Pública de México. De acuerdo con sus recomendaciones, sólo se entrevistó a los profesionales de salud que accedieron voluntariamente a participar del estudio y en todos los casos se obtuvo el consentimiento informado de los individuos entrevistados y se les garantizó el anonimato. Las entrevistas fueron realizadas en el lugar de trabajo, en el espacio privado de un cubículo o consultorio.

\section{Resultados}

El análisis de las entrevistas realizadas a médicos/as y enfermeros/as deja en evidencia que en el plano de las prácticas en los servicios de salud con mujeres víctimas de violencia no existen grandes discrepancias entre los prestadores de salud entrevistados. La atención a la violencia resulta en general inadecuada, tal como lo reportan otros estudios realizados en el país 9,10,11 y entre sus prácticas habituales encontramos que: - Los prestadores no se guían por lo que la NOM-190 prescribe como rutina obligatoria en estos casos y esto obedece a que el conocimiento de esta norma es deficiente o nulo;

- Sólo unos pocos profesionales entrevistados reportaron haber recibido una mínima ca- pacitación en el tema, y ésta consistió en sólo unas pláticas de asistencia voluntaria;

- Los prestadores de la salud suelen utilizar formularios de registro de accidentes para reportar lesiones y no consignan en ellos que éstas fueron originadas por violencia doméstica. Esto obedece a que los formularios no siempre están disponibles en los servicios de salud;

- En general la violencia suele pasarse por alto, y cuando es detectada, la mujer que la reporta expresamente es canalizada al servicio de trabajo social o de psicología de la institución. En la mayoría de los casos, salvo excepciones significativas, no existe un seguimiento posterior.

Si bien en el nivel de atención no se observan mayores diferencias en las prácticas más habituales, sí se observan variaciones en cuanto a la percepción subjetiva de los prestadores de salud ante el problema de la violencia y sus explicaciones del fenómeno. Hemos identificado diferentes argumentos y significados en torno a la violencia contra las mujeres, a los que hemos clasificado en cuatro tipos diferentes de "discursos sobre la violencia". Esta tipología se construyó tomando en cuenta dos grandes matrices valorativas, según predominaran en ellos valores tradicionales o modernos con respecto al género, y las combinamos con tres aspectos diferenciales expresados por los prestadores de salud: su percepción de la violencia en las pacientes, su disposición hacia la atención de las mismas, y sus prácticas más frecuentes. Los tipos resultantes fueron: (1) discurso dominante basado en estereotipos rígidos de género; (2) anti-violencia basado en valores tradicionales de género; (3) anti-violencia basado en ideas sobre "lo moderno"; y (4) anti-violencia basado en los derechos de la mujer. Para un análisis de esta tipología nos remitimos a Herrera et al. 12 .

Un primer discurso lo ubicamos en los prestadores que compartían la idea de que el problema de la violencia contra las mujeres es inexistente, repitiendo con frecuencia la frase: "aquí no se ven casos". Cuando se les mencionaban directamente las altas cifras de prevalencia de violencia en el país y en el estado donde ellos mismos trabajaban, ofrecían diferentes argumentos. Algunos de ellos tendían a minimizar el problema o a diluir su importancia comparándolo con otro tipo de problemas tales como la violencia contra los niños y contra los ancianos, según ellos, de igual magnitud. Otros entrevistados enjuiciaban a las víctimas de la violencia o las hacían responsables del maltrato que sufren. En casos extremos mostraban desprecio o se burlaban de quienes sufren violencia: "Bueno, es que también nada más estamos hablando de mujeres. ¡También hay violencia 
contra los hombres! (...) También hay hombres que sufren, sufren violencia por parte de las mujeres. (...) Todo es sobre la mujer, se la protege (...), a ver: ¿por qué no se enfocan también en los padres solteros?” (Médico de 41 años de la ciudad de México); “Sí, observamos que la mamá de los niños pues los abandona, los deja con la abuela o la tía, ella vuelve a hacer su vida, se va con su pareja, regresa y como que los niños están descuidados" (Enfermera de 45 años de la ciudad de México).

Con estos argumentos los prestadores quedan exentos de cualquier responsabilidad en cuanto a su atención como profesionales de salud y prevalece la ignorancia directa, o bien la aceptación pasiva de la violencia como algo "natural". Si hay alguna lesión, ésta es tratada con los procedimientos de rutina y no se indaga en sus causas ni se da seguimiento. Este grupo de prestadores no está dispuesto a involucrarse en cuestiones legales. Al respecto una enfermera respondió: "Lo que pasa es que luego se mete uno en muchos problemas. (...) Sí, evitar meterse en cosas más profundas (...) que a lo mejor a la salida lo vayan a amenazar a uno o algo" (Enfermera de 43 años de la ciudad de México).

Esta disposición de los profesionales de la salud hacia la violencia puede ser potencialmente perjudicial, y esto ha sido observado en mujeres que reportan haber sido doblemente victimizadas: por parte del abusador, y también por parte del personal de salud, que las responsabiliza a ellas del maltrato sufrido. Similares resultados se observan en otros estudios a nivel internacional 13,14,15.

Un segundo discurso, al que adhieren exclusivamente prestadoras de salud de sexo femenino, expresaron compasión e identificación con las mujeres víctimas, con base en valores y normas morales tradicionales. La violencia es vista como una desviación indeseable de los ideales de la armonía familiar, basada en los roles y virtudes tradicionalmente asignados a cada sexo. La mujer debe sobreponerse a su situación en pos de cumplir su responsabilidad y dedicación a los demás, en particular a sus hijos: "Ya cuando se toma, digamos, confianza, nosotros le decimos: 'señora, pero por favor, su dignidad está ante todo, tiene sus hijos, ahorita tuvo un golpe digamos leve, va a recibir más golpes, tiene a sus niños, despierte'. (...) Entonces prácticamente le digo yo a ella, 'tienes que tener mucha fuerza de voluntad para poder salir, quererse un poquito para tener conciencia de esa situación y sacar a tus hijos, porque finalmente son los más dañados" (Enfermera de 42 años de Saltillo).
Las mujeres que sostienen este tipo de discurso manifestaron, asimismo, sentimientos tales como vergüenza de hablar del tema con sus pacientes, y la renuncia a inmiscuirse en un asunto que consideran doloroso, pero por sobre todo privado. Consideramos que en estos casos se trata de un fenómeno de identificación empática femenina de estas profesionales con sus pacientes, y que se hace evidente en el temor a los hombres violentos o misóginos, a las represalias del agresor, o al ser mal vistas por sus colegas masculinos: “...si a pesar de todo tú me dices, 'doctora, quiero seguir con mi marido porque lo amo, por mis hijos, por la sociedad, por esto o aquello', estás en derecho a hacerlo y que nadie te diga absolutamente nada, ni yo, que me lo acabas de contar, porque te digo que (...) porque si no el día de mañana tendría problemas en cuestiones de que la familia vendría a lo mejor a reclamarme..." (Médica de 36 años de la ciudad de México).

Las profesionales entrevistadas que se adhieren a este discurso suelen mostrarse sensibles al problema y acceden a hablar con la mujer maltratada cuando esta demanda ayuda de manera explícita, darle consejos "de mujer a mujer" y ofrecerles información sobre lugares especializados o canalizándola a trabajo social para que ahí se la proporcionen.

Un tercer discurso está conformado por opiniones contrarias a la violencia, pero también ciertos prejuicios sociales acerca de quienes la padecen o la ejercen, a quienes suelen identificar con lo "atrasado". Los prestadores de salud que sostienen este discurso, asocian la violencia con la falta de cultura y educación, con la pobreza o el origen rural o indígena: "[el problema] viene sobre todo en la escolaridad, la persona que tiene escolaridad baja y sobre todo si vivió en una familia que sus papás golpean, eso lo toman como un trato de conducta normal" (Médica de 57 años de la ciudad de México).

"Son gentes de bajos recursos, con muchos problemas sociales y psicológicos (...) o traen a sus hijos porque son madres solteras o divorciadas (...) gente sin empleo o gentes que vienen a la aventura (...) gente del área rural, gente agresiva, y si lo vemos, son lugares donde hay un alto índice de criminalidad..." (Médico de 47 años de Chetumal).

El sentimiento hacia las mujeres maltratadas más frecuentemente expresado por estos prestadores de salud fue de indignación hacia la víctima, por no saber hacerse respetar y dejarse maltratar, pero no así hacia el agresor, quien permanece invisible en el discurso, a quien no condenan moralmente o no reconocen como el verdadero victimario. El lenguaje utilizado 
para referirse al problema de la violencia encubre una actitud de desprecio social hacia las víctimas de la violencia y su entorno.

Los prestadores de salud que ubicamos como portadores de este discurso se mostraron ambivalentes sobre el problema: por un lado lo consideraban grave, pero por otro, aducían cuestiones de educación y status social para justificar su distancia con respecto a las personas que "son violentos o sufren violencia".

"Pues siento tristeza, ¿no?, siento pues que, bueno a la mejor me proyecto como mujer y no me gustaría, pero, pero le vuelvo a decir, son sentimientos encontrados porque a ellas les gusta, o ellas lo toleran, ¿no?, porque por ejemplo. (...) Tuve una compañera hace como dos, tres años, pues mi compañera como de cuarenta y tantos años y tenía una pareja de, de 25, le dieron una golpiza y, y bueno me causó mucha admiración porque nosotros somos profesionistas, porque tenemos un nivel escolar, porque somos autosuficientes económicamente" (Médica de 45 años de ciudad de México).

Finalmente, ubicamos un último tipo de discurso al que se adhieren en su mayoría profesionales de servicios de urgencias. Estos expresaban un claro rechazo a la violencia de pareja por considerarla un delito y una violación de los derechos humanos, e identificaban al machismo y a la desigualdad de poder entre los géneros como la causa de la violencia contra la mujer: “Bueno, no me gusta la, la injusticia ni la (...) ora sí que la discriminación en (...) porque uno es mujer, ¿verdad?, lleva uno tanto los derechos, o sea lleva la igualdad, entonces no, no me da temor el preguntar si fue golpeada, como le digo, ya ellas mismas empiezan a pues a platicar, y muchas veces ya hasta ellas: 'no, pues mi esposo...'o, o ya hasta nos dan a saber su vida hasta privada, ¿verdad?, pero no, no me es difícil" (Enfermera de 35 años de Saltillo).

El conjunto de disposiciones de este grupo de profesionales, al que podríamos caracterizar como el más conciente del problema, evidencia indignación ante lo que consideran una injusticia y una alta predisposición a atender los casos, con diversos grados de involucramiento personal: desde dar consejos hasta acompañar a la mujer a denunciar ante el Ministerio Público. Buscan que la mujer adquiera capacidades y libertades para actuar, pero muchas veces sienten impotencia ante los magros resultados obtenidos, tanto por falta de apoyo institucional como por las dificultades de las propias mujeres para salir del círculo de la violencia, en contextos sociales poco favorables a ello.

"Pues nosotros le damos la atención medica ¿verdad? Si percibimos que se requiere, la cana- lizamos al médico familiar y para apoyo de psi-

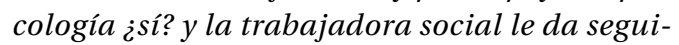
miento al caso, toma datos (...) y al ministerio público (...) O ya si la paciente dice, o sea acusa y a a alguien, la orienta para que vaya a poner su denuncia, o sea ella le dice a dónde tiene que acudir y eso (...) se supone que viene el médico, de hecho muchas veces nos ha tocado, viene el medico legista y nos interroga acerca de las lesiones, toma los datos (...) y habla con la familia y toma datos del expediente y habla con el$l a "$ (Médica de 37 años de Saltillo).

"Pues en este caso, sí, que la Institución también tuviera la apertura, esa elasticidad, ese apoyo para que el médico pueda (...) salir y atestiguar, porque pues, está bien hacerlo, porque debemos de apoyar a la persona que viene buscando ayuda ¿no? Yo pienso que sí, como personas, como medico familiar debemos participar también en esos eventos, claro que siempre apoyados por la institución, porque aquí no podemos..." (Médico de 40 años de Chetumal).

Resulta evidente que el repertorio de significados sociales atribuidos a la violencia contra las mujeres de parte de los prestadores de salud entrevistados no es sino un reflejo de los diferentes valores y normas sociales que pautan la relación entre los sexos en la sociedad de la que ellos mismos provienen. Kim \& Motsei 16, como también Gerbert et al. 17 han mostrado que dado que el personal de salud es producto de la misma tradición cultural que las pacientes, no necesariamente juzga negativamente los valores y normas tradicionales que dan soporte al abuso contra la mujer. Por ello, es indudable que la sensibilidad y la atención adecuada de la violencia doméstica en el ámbito de la salud, tal como lo prevé la NOM-190, dependen de un programa de capacitación y sensibilización amplio de los prestadores de salud que permita cuestionar los valores tradicionales de la cultura patriarcal que favorecen la tolerancia o justificación de la violencia masculina.

\section{Discusión}

En el plano internacional, son escasos los estudios que se dedican a evaluar el impacto de las políticas y programas de salud destinados a resolver el problema de la violencia doméstica, tal como lo señalan Glowa et al. 18, Gerbert et al. 19, Chang et al. 20 y Fogarty et al. 21. En consecuencia, como apunta Cole 22 , no hay todavía un acuerdo claro sobre cuál es el mejor modelo de atención a seguir. En México, la problemática de la violencia contra las mujeres como objeto de políticas de salud pública, es aún mas 
reciente, y existen por tanto pocos estudios de impacto de los programas, así como evaluaciones de su viabilidad, en términos de conocer las barreras que pueden enfrentar los encargados de implementar tales políticas. En particular, la ENVIM 5 ha puesto en evidencia la insuficiencia en los programas gubernamentales nacionales para poder capacitar e implementar la normatividad oficial (recomendaciones y procedimientos frente a los casos de violencia contra las mujeres), ya que la mayoría de los prestadores desconocen su existencia.

Estudios realizados en contextos con muy diferentes marcos institucionales y culturales 23 , $24,25,26$ han señalado los diversos límites que enfrentan los prestadores de salud para identificar la violencia doméstica en sus pacientes, incluso en aquellos que están comprometidos a hacerlo.

Una revisión de la literatura sobre el tema, así como el análisis de nuestros datos nos permite distinguir limitaciones provenientes de diferentes ámbitos y que resultan en una atención deficiente de los casos de violencia. Entre estas limitaciones identificamos, (a) las concepciones que provienen de la cultura, (b) ciertas barreras de la misma práctica profesional, (c) problemas en la interacción con el paciente y (d) limitaciones provenientes de la relación con el subsistema jurídico.

En el ámbito de las concepciones que provienen de la tradición cultural hemos podido identificar que una mala atención al problema de la violencia se expresa en prácticas muy perjudiciales tales como culpabilizar a la paciente, a quien se hace responsable del maltrato de que resulta objeto; ignorar el problema que es considerado un fenómeno natural; manifestar vergüenza o temor a tocar un tema privado; y desprecio y prejuicios sociales.

Respecto de ciertas limitaciones que provienen de la práctica profesional, algunos de nuestros entrevistados reportan no tener el tiempo suficiente para darle una atención adecuada a estas pacientes, coincidiendo así con los resultados arrojados por Sugg \& Inui 27 que en un estudio entre médicos de primer nivel de atención en Estados Unidos, por ejemplo, encontró como barreras para una adecuada atención a la violencia la falta de entrenamiento y la falta de tiempo.

Asimismo, Gerbert et al. 28 identifican como barreras el olvido de que algunas constelaciones de síntomas pueden ser consecuencia indirecta de la violencia, entre ellas: eventos pasados de abuso físico, sexual o emocional, depresión, ansiedad, migraña persistente, dolor pélvico, dolor abdominal vago que no cede an- te el tratamiento, discrepancia entre la descripción que hace la paciente del modo en que se produjo la lesión y lo que el médico observa, "propensión al accidente" en algunas pacientes, marcas en lugares extraños, etc.

En el ámbito de la interacción paciente-médico se observan limitaciones de diferentes tipos. Gerbert et al. 28 señalan lo fatigante y poco satisfactorio que es para el personal de salud tratar de resolver estos casos, debido a la reticencia de las pacientes a revelar la violencia que sufren. Un elemento importante que observan los médicos, según Booher et al. 29 y Gerbert et al. 28 , es que han sido entrenados para identificar rápido el problema y "arreglarlo", por lo que se inclinan más a "arrancar una confesión" a las mujeres con fines de diagnóstico y tratamiento, que a elaborar una estrategia de acercamiento más lento y sensible, que a la larga daría mejores resultados. La falta de resultados visibles y rápidos de la primera estrategia, a su vez desmotiva a los prestadores de salud. También la falta de tiempo para establecer una relación de confianza que permita involucrarse, sin que esto resulte amenazante para la paciente, resulta en una limitación importante, según Chang et al. 20.

Un dato importante que surge de la ENVIM 5 es que las mujeres que sufren violencia no acuden a los servicios de salud para atender las lesiones causadas por la misma, y menos aún a levantar denuncias ante instancias de justicia. Los factores que contribuyen a esta inacción son múltiples, entre ellos se ubican el estigma y discriminación de que son objeto (o que temen sufrir) por parte de los funcionarios que las atienden en esas instancias. A pesar de ello, las mujeres sí acuden a los servicios por otros problemas de salud, muchos de los cuales son consecuencia indirecta de situaciones de violencia.

Estudios a nivel internacional con mujeres maltratadas como el de Rodríguez et al. $30 \mathrm{y}$ Zink et al. 31 muestran que estas mujeres estarían dispuestas a hablar de la violencia si los prestadores de salud se mostraran más sensibles. A pesar de que en algunos casos ni siquiera en estas condiciones revelan la situación en la que se encuentran, en general, puede decirse que la percepción de las mujeres es que los profesionales de la salud dan una respuesta inapropiada al problema de la violencia doméstica que sufren, tal como señalan Naumann et al. 32 .

Las limitaciones que provienen de la relación con el subsistema jurídico se observa en nuestro estudio como una manifiesta desconfianza en las instituciones judiciales. Chang et al. ${ }^{20}$ por su parte señalan también que la obli- 
gación de reportar a la justicia puede ser un desincentivo para que los prestadores de salud se ocupen del problema, por preocupaciones acerca del bienestar y confidencialidad de las pacientes.

Los criterios que pautan el desempeño profesional del personal de salud provienen de un sistema organizacional que responde en teoría al Estado, y establece ciertas obligaciones para los prestadores en tanto representantes de las políticas gubernamentales. En este caso, se trata de su obligación de identificar, atender y reportar como delito casos de violencia detectados entre las pacientes.

En la práctica, este grupo de profesionales está formado por individuos inmersos en un mundo de patrones culturales que dan sustento a la misoginia. No solamente se ha constatado que un cierto porcentaje de ellos está involucrado en situaciones de violencia doméstica, sino que la mayoría comparte los valores sociales que contribuyen a "tolerar" el problema o a concebirlo como algo privado, en lo que no se debe intervenir.

Se hace así evidente que los prestadores de salud se encuentran en un lugar de tensión, donde confluyen de manera conflictiva discursos e imaginarios sobre lo público y lo privado, sobre la familia, las normas de género y la autoridad, y en particular sobre la violencia intrafamiliar, y que resultan de un proceso amplio de socialización, educación y formación profesional.

Las posiciones simultáneas que ocupan los profesionales de salud como prestadores de salud, como funcionarios del Estado, como agentes sociales nos permiten caracterizar el problema de la atención de la violencia como dilemático. Muchos de estos prestadores encuentran dificultades para concebir a las víctimas de violencia como sujetos de derechos y no como "pacientitas" (una manera muy común entre los médicos en México de referirse a su clientela, en especial la femenina), y más aún para concebir su papel fuera de los límites estrictos de lo que consideran debe ser la práctica médica (curar lesiones y enfermedades del cuerpo de manera rápida y eficaz).

Si bien en varios de los casos los discursos justificativos de la inacción frente a la violencia en los servicios, probablemente, refleje una indiferencia ante el problema, creemos que en la mayoría de los profesionales estos mismos discursos podrían ser más bien mecanismos de "distanciamiento", para evitar la frustración frente a un problema con el cual no se sienten preparados para lidiar, dada la escasez de resultados tangibles.

\section{Conclusiones}

Las dificultades para una adecuada atención al problema de la violencia doméstica en los servicios de salud y para hacer operativa la NOM190 , como puede advertirse, forman un entramado complejo de problemas, tanto institucionales, como culturales y sociales.

Hemos identificado entre los prestadores de salud entrevistados un repertorio de significados sociales sobre la violencia, que interviene directamente en el tipo de atención brindada a las víctimas. Estas concepciones sobre la violencia masculina provienen de una tradición cultural de la que los profesionales forman parte.

Paralelamente a la difusión de una cultura de derechos y de equidad de género, deben promoverse los mecanismos institucionales y organizacionales necesarios para que estos valores vayan encarnando en las instituciones y en su personal, pero desde una comprensión más profunda de la subjetividad de estos individuos, de sus prácticas profesionales y de los repertorios de significados sociales que utilizan, conciente o inconscientemente, para dar sentido a una realidad cotidiana difícil de manejar.

\section{Resumen}

Se realizó un estudio cualitativo con 30 médicas/os y enfermeras/os del sector público en México, con el objetivo de conocer en profundidad las concepciones $y$ prácticas frente a la violencia doméstica. Una encuesta reciente puso en evidencia que la mayoría de los profesionales de salud no conocen la Norma Oficial Mexicana que establece los criterios de atención de estos casos. Los resultados de las entrevistas evidencian que no existen diferencias entre los profesionales a nivel de la atención, y que además la atención resulta inadecuada. Por otra parte, hemos constatado la existencia de un repertorio de significados sociales en torno a la violencia que provienen de la tradición cultural y que dificultan una adecuada atención a este problema.

Violencia Doméstica; Personal de Salud; Violencia Contra la Mujer 


\section{Colaboradores}

C. Herrera coordinó, diseñó y participó en todas las etapas de la investigación. C. Agoff participó en el diseño de la investigación, en el trabajo de recolección de los datos y supervisó el análisis de las entrevistas. Ambas participaron en la escritura del presente artículo.

\section{Referencias}

1. Asamblea General, Organización de las Naciones Unidas. Declaración sobre la eliminación de la violencia contra la mujer. Resolución de la Asamblea General 48/104 del 20 de diciembre de 1993. Nueva York: Organización de las Naciones Unidas; 1993.

2. Organización Panamericana de la Salud/Organización Mundial de la Salud. Informe mundial sobre la violencia y la salud. http:/ / www.paho.org/ Spanish/AM/PUB/introduccion.pdf (accedido el 12/Jul/2005).

3. Organización de las Naciones Unidas. Programa de acción: Conferencia Internacional sobre Población y Desarrollo. El Cairo: Organización de las Naciones Unidas; 1995.

4. Organización de las Naciones Unidas. Declaración y plataforma de acción: Cuarta Conferencia Mundial sobre la Mujer. Pekín: Organización de las Naciones Unidas; 1995.

5. Secretaría de Salud/Instituto Nacional de Salud Pública. Encuesta nacional de violencia contra las mujeres (ENVIM). Cuernavaca: Instituto Nacional de Salud Pública; 2003.

6. Secretaría de Salud. Norma oficial mexicana, NOM-190-SSA1-1999, prestación de servicios de salud. Criterios para la atención de la violencia familiar. México DF: Secretaría de Salud; 1999.

7. Glaser B, Strauss A. The discovery of grounded theory. Strategies for qualitative research. Chicago: Aldine; 1967.

8. Strauss A, Corbin J. Basics of qualitative research. Newbury Park: Aldine; 1990.

9. Méndez-Hernández P, Valdez-Santiago R, Viniegra-Velázquez L, Rivera-Rivera L, Salmerón-Castro J. Violencia contra la mujer: conocimiento y actitud del personal médico del Instituto Mexicano del Seguro Social, Morelos, México. Salud Pública Mex 2003; 5:472-82.

10. Secretaría de Salud/Instituto Nacional de Salud Pública. Violencia contra las mujeres. Un reto para la salud pública en México. Informe ejecutivo de la Encuesta Nacional de Violencia contra las Mujeres. Cuernavaca: Instituto Nacional de Salud Pública; 2004.
11. Fawcett G. Manejo de víctimas de violencia doméstica: desarrollo y evaluación de un programa dirigido al personal de salud. México DF: Population Council/INOPAL III; 1998 (Documentos de Trabajo, 26).

12. Herrera C, Rajsbaum A, Agoff C, Franco A. Entre la negación y la impotencia: prestadores de servicios de salud ante la violencia contra las mujeres en México. Salud Publica Mex; en prensa.

13. Sampselle CM. The role of nursing in preventing violence against women. J Obstet Gynecol Neonatal Nurs 1991; 20:481-7.

14. Langford DR. Policy issues for improving institutional response to domestic violence. J Nurs Adm 1996; 26:39-45.

15. Campbell JC. Battered women's experiences in the emergency department. J Emerg Nurs 1994; 20:280-8.

16. Kim J, Motsei M. "Women enjoy punishment": attitudes and experiences of gender-based violence among PHC nurses in rural South Africa. Soc Sci Med 2002; 54:1243-54.

17. Gerbert B, Gansky S, Tang J, McPhee S, Carlton $\mathrm{R}$, Herzig K, et al. Domestic violence compared to other health risks. A survey of physicians' beliefs and behaviors. Am J Prev Med 2002; 23:8290.

18. Glowa P, Frasier P, Wang L, Eaker K, Osterling W. What happens after we identify intimate partner violence? The family physician's perspective. Fam Med 2003; 35:730-6.

19. Gerbert B, Caspers N, Milliken N, Berlin M, Bronstone A, Moe J. Interventions that help victims of domestic violence. J Fam Pract 2000; 49:889-95.

20. Chang J, Cluss P, Ranieri LA, Hawker L, Buranosky $\mathrm{R}$, Dado D, et al. Health care interventions for intimate partner violence: what women want? Womens Health Issues 2005; 15:21-30.

21. Fogarty C, Burge S, McCord E. Communicating with patients about intimate partner violence: screening and interviewing approaches. Fam Med 2002; 34:369-75.

22. Cole T. Is domestic violence screening helpful? JAMA 2000; 284:551-7. 
23. Family Violence Prevention Project. An informational packet for health professionals. San Francisco: Family Violence Prevention Fund; 1993.

24. Silva IV. Violência contra mulheres: a experiência de usuárias de um serviço de urgência e emergência de Salvador, Bahia, Brasil. Cad Saúde Pública 2003; 19 Suppl 2:S263-72.

25. Zink T, Elder N, Jacobson J, Klostermann B. Medical management of intimate partner violence considering the stages of change: precontemplation and contemplation. Ann Fam Med 2004; 2:231-9.

26. Taft A, Broom D, Legge D. General practitioner management of intimate partner abuse and the whole family: qualitative study. BMJ 2004; 328:618.

27. Sugg $N$, Inui T. Primary care physicians' response to domestic violence. Opening Pandora's box. JAMA 1992; 267:3157-60.
28. Gerbert B, Caspers N, Bronstone A, Moe J, Abercrombie P. A qualitative analysis on how physicians with expertise in domestic violence approach the identification of victims. Ann Intern Med $1999 ; 131: 578-84$

29. Booher T, Lane J, Davis L. Domestic violence in the outpatient setting. J Am Acad Dermatol 2003; 50:262-5.

30. Rodríguez H, Bauer M, Flores-Ortiz Y, SkupinskiQuiroga S. Factors affecting patient-physician communication for abused Latina and Asian immigrant women. J Fam Pract 1998; 47:309.

31. Zink T, Regan S, Goldenhar L, Pabst S, Rinto B. Intimate partner violence: what are physicians' perceptions? J Am Board Fam Pract 2004; 17:332-40.

32. Naumann P, Langford D, Torres S, Campbell J, Glass N. Women battering in primary care practice. Fam Pract 1999; 16:343-52.

Recibido el 19/Jul/2005

Versión final presentada el 14/Dic/2005

Aprobado el 17/Mar/2006 